\title{
Interactive comment on "Ensemble-based statistical interpolation with Gaussian anamorphosis for the spatial analysis of precipitation" by Cristian Lussana et al.
}

Alberto Carrassi (Editor)

n.a.carrassi@reading.ac.uk

Received and published: 25 September 2020

Dear Authors,

I found your answers to the Reviewers exhaustive and I invite you to submit a revised version of the manuscript with a "diff-version" included.

Best Regards

Alberto Carrassi 
Interactive comment 